\title{
Contrasting Roles of Basolateral Amygdala and Orbitofrontal Cortex in Impulsive Choice
}

\author{
Catharine A. Winstanley, David E. H. Theobald, Rudolf N. Cardinal, and Trevor W. Robbins \\ Department of Experimental Psychology, University of Cambridge, Downing Site, Cambridge CB2 3EB, United Kingdom
}

\begin{abstract}
The orbitofrontal cortex (OFC) and basolateral nucleus of the amygdala (BLA) share many reciprocal connections, and a functional interaction between these regions is important in controlling goal-directed behavior. However, their relative roles have proved hard to dissociate. Although injury to these brain regions can cause similar effects, it has been suggested that the resulting impairments arise through damage to different, yet converging, cognitive processes. Patients with OFC or amygdala lesions exhibit maladaptive decision making and aberrant social behavior often described as impulsive. Impulsive choice may be measured in both humans and rodents by evaluating intolerance to delay of reinforcement. Rats with excitotoxic lesions of the BLA and OFC were tested on such a delay-discounting procedure. Although lesions of the BLA increased choice of the small immediate reward, indicating greater impulsivity, OFC lesions had the opposite effect, increasing preference for the larger but delayed reward. The fact that the delay did not devalue the large reward to such an extent in OFC-lesioned animals supports the suggestion that the OFC is involved in updating the incentive value of outcomes in response to devaluation. In contrast, the BLA-lesioned animals markedly decreased their preference for the large reward when it was delayed, potentially because of an inability to maintain a representation of the reward in its absence. This is the first time that lesions to these two structures have produced opposite behavioral effects, indicating their distinct contributions to cognition.
\end{abstract}

Key words: amygdala; cognitive; frontal; impulsivity; goal directed; delay discounting

\section{Introduction}

The orbitofrontal cortex (OFC), encompassing ventrolateral and ventromedial frontal cortex, is integral to various corticocortical networks implicated in higher-order cognition, receiving polymodal input from sensory cortices as well as interacting with subcortical areas including the mediodorsal thalamus (Nauta, 1971; Öngür and Price, 2000). In particular, the OFC shares many reciprocal connections with the basolateral nucleus of the amygdala (BLA) (Krettek and Price, 1977; McDonald, 1991), one of the larger amygdaloid nuclei, which interconnects with temporal lobe structures such as the hippocampus as well as cortical and striatal regions (Pitkänen, 2000). OFC and BLA lesions often cause similar behavioral effects (Schoenbaum et al., 2003a), a pattern attributed to the extensive connections linking these regions. Dissociating the roles these structures play in goal-directed behavior has therefore proved difficult.

The OFC and BLA are important nodes in the limbic corticostriatal loop, linking them to other structures involved in reward learning, including the nucleus accumbens (NAC) (Cardinal et

Received Dec. 19, 2003; revised April 7, 2004; accepted April 11, 2004.

This work was supported by a Wellcome Trust Programme grant and completed within the Medical Research Council (MRC) Centre for Behavioral and Clinical Neuroscience. C.A.W. was supported by an MRC studentship. We are grateful to Yogita Chudasama and Jonathan Lee regarding histological verification of the lesions.

Correspondence should be addressed to Catharine Winstanley, Department of Psychiatry, University of Texas Southwestern Medical Center, Dallas, TX 75390-9070. E-mail: cath.winstanley@utsouthwestern.edu. DOI:10.1523/JNEUROSCI.5606-03.2004

Copyright $\odot 2004$ Society for Neuroscience $\quad$ 0270-6474/04/244718-05\$15.00/0 al., 2002). NAC lesions increase impulsivity in rats on a delay-togratification task, in which impulsive choice is defined as the selection of a small immediate over a larger delayed reward (Ainslie, 1975; Logue, 1988; Evenden and Ryan, 1996; Cardinal et al., 2001). Impulsivity is a core deficit in neuropsychiatric illnesses, such as attention deficit hyperactivity disorder, and rodent delayto-gratification tasks have been used to investigate the pharmacology of impulsive behavior (Evenden and Ryan, 1996). However, structures conveying information to the NAC necessary for appropriate response selection are unknown. Lesions of prelimbic (PrL) or anterior cingulate (ACC) cortex cause little or no change in impulsive choice (Cardinal et al., 2001) despite strong interconnections with the NAC.

Unlike patients with damage to dorsolateral prefrontal cortex (PFC), patients with OFC and amygdala lesions exhibit patterns of behavior often described as impulsive as exemplified by their performance on gambling tasks (Damasio, 1994; Bechara et al., 1998, 1999; Rogers et al., 1999). Although healthy volunteers select options leading to long-term gain, patients with OFC or amygdala lesions make "risky" choices, preferring options associated with large short-term rewards but long-term loss. However, the behavior of OFC patients can suggest paradoxically reduced impulsivity. Their risky decision making can be very slow, contradicting the impulsive trait of making over-hasty judgements (Damasio, 1994; Rogers et al., 1999). Although patients with OFC damage generate appropriate autonomic responses to affective stimuli and to losing money in the gambling paradigm, 
patients with amygdala lesions do not (Bechara et al., 1999). The amygdala plays an important role in processing emotionally significant stimuli (LeDoux, 2000), which can guide goal-directed behavior (Gallagher, 2000). For example, BLA lesions profoundly impair fear conditioning to a discrete conditioned stimulus (CS), yet less is known about the role of the OFC in such processes. However, lesions to both structures disrupt the utilization of CSs in goal seeking (Gallagher, 2000). We therefore postulated that BLA and OFC lesions would alter behavior in a rodent model of impulsive choice, and that this paradigm could reveal dissociable deficits after damage to these areas.

\section{Materials and Methods}

Subjects. Subjects were 32 male Lister Hooded rats (Charles River, Kent, UK) weighing 300-320 gm at the start of each experiment and maintained at $85 \%$ of their free-feeding weight. Water was available ad libitum. Animals were housed in groups of four under a reverse light cycle (lights on from 7:00 P.M. to 7:00 A.M.). Testing took place between 8:00 A.M. and 7:00 P.M. 5-6 d per week. Experiments were undertaken in accordance with the UK Animals (Scientific Procedures) Act 1986.

Behavioral testing. The behavioral apparatus and testing procedure have been described previously in detail (Cardinal et al., 2000). Testing occurred in eight operant conditioning chambers (Med Associates, Georgia, VT) fitted with two retractable levers located on either side of a food magazine, into which $45 \mathrm{mg}$ of food pellets (Noyes dustless pellets; Sandown Scientific, Middlesex, UK) could be delivered, a magazine light, and a houselight. Magazine entry was detected by an infrared photocell beam located across the entrance. Each chamber was encased in a soundattenuating box and fan ventilated. The apparatus was controlled by software written in Arachnid running on Acorn Archimedes Series computers (Cambridge, UK).

Subjects first learned to lever-press for food reward and to nose-poke in the magazine to trigger presentation of the levers. Animals were then trained to perform a delay-discounting task until stable baseline behavior was achieved (Fig. 1). Each session lasted $100 \mathrm{~min}$ and consisted of 5 blocks of 12 trials. Each trial lasted $100 \mathrm{sec}$ regardless of the choice made by the subject, and each block began with two forced-choice trials. The onset of the houselight signaled the beginning of each trial, in which the rat had to nose-poke in the magazine to trigger lever presentation. An omission was scored if the rat failed to respond at the food magazine or subsequently on the levers within $10 \mathrm{sec}$, and the box returned to the inter-trial interval (ITI) state with the houselight extinguished until the next trial was scheduled to begin. The houselight was extinguished and the levers retracted once a response was made. Responding on one lever (lever A) always provided a small immediate reward of one pellet, the other (lever B) a large reward of four pellets. The location of levers A and $\mathrm{B}$ were counterbalanced between subjects. As the session progressed, the delay to the large reward increased in each block of trials from 0 to 10, 20, 40 , and then $60 \mathrm{sec}$. Onset of the traylight signaled food delivery, after which the box returned to the ITI state.

Surgery. Subjects were divided into three groups, matched for baseline performance, and received either bilateral lesions of the OFC $(n=12)$, BLA $(n=10)$, or vehicle infusions $(n=10)$ into one of these areas. Subjects were anesthetized using $100 \mathrm{ml} / \mathrm{kg}$ Avertin [10 gm of 2,2,2tribromoethanol (Sigma, Poole, UK) in 5 gm of tertiary amyl alcohol, diluted in a solution of $40 \mathrm{ml}$ of ethanol and $450 \mathrm{ml}$ of PBS] and secured in a stereotaxic frame. Excitotoxic lesions were made using $0.09 \mathrm{M}$ quinolinic acid dissolved in $0.1 \mathrm{~m}$ phosphate buffer, and the $\mathrm{pH}$ was adjusted with $0.1 \mathrm{M} \mathrm{NaOH}$ to between 6 and 7. Infusions of toxin-vehicle were made using a 31 gauge stainless steel injector (Coopers Needleworks, Cambridge UK) attached to a Hamilton (Reno, NV) microinfusion pump by polyethylene tubing (Portex, Hythe, UK) according to the following coordinates (Paxinos and Watson, 1998): BLA: site 1 anteroposterior (AP), -3.0 , lateral (L), \pm 4.6 , dorsoventral (DV), $-7.8,0.1 \mu \mathrm{l}$ infused over $2 \mathrm{~min}$, site $2 \mathrm{AP}-2.8, \mathrm{~L}, \pm 4.6, \mathrm{DV},-7.8,0.2 \mu \mathrm{l}$ per $2 \mathrm{~min}$; OFC: site $1 \mathrm{AP},+4.0, \mathrm{~L}, \pm 0.8, \mathrm{DV},-3.4,0.2 \mu \mathrm{l}$ per $3 \mathrm{~min}$, site $2 \mathrm{AP},+3.7$, $\mathrm{L}, \pm 2.0, \mathrm{DV},-3.6,0.3 \mu \mathrm{l}$ per $3 \mathrm{~min}$, site $3 \mathrm{AP}+3.2, \mathrm{~L}, \pm 2.6, \mathrm{DV},-4.4$,
A

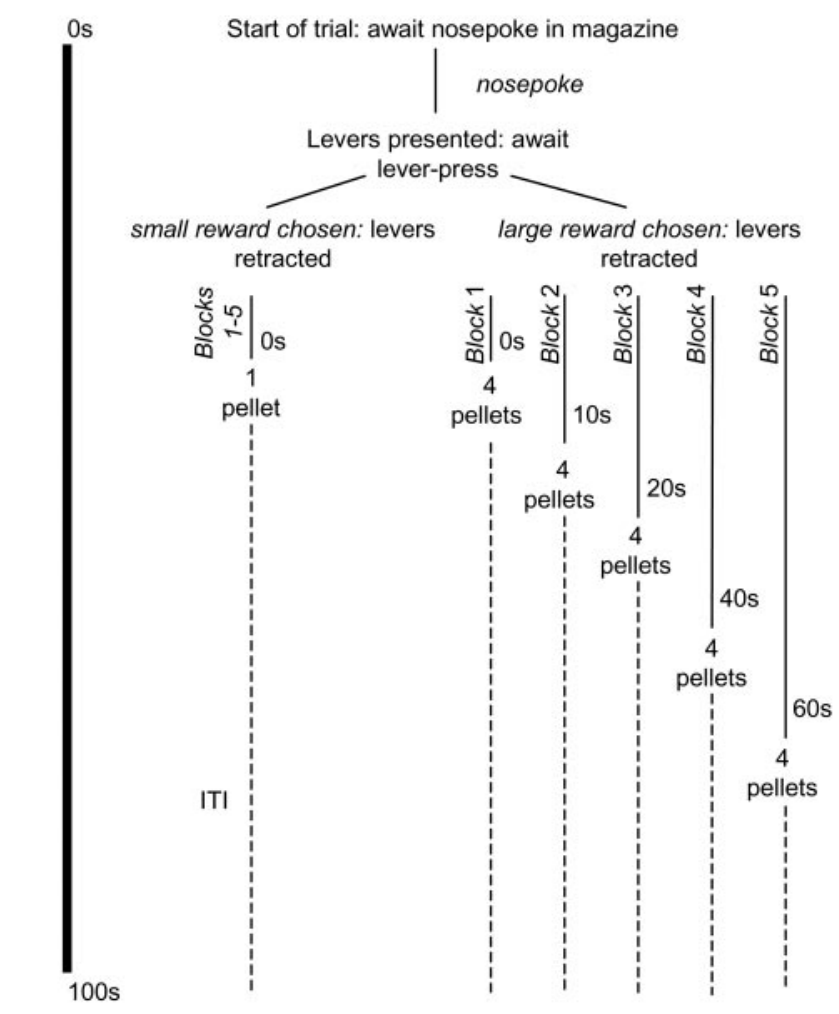

B

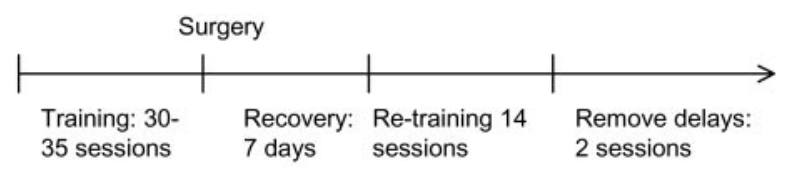

Figure 1. $A, B$, Basic outline of the delay-discounting task in terms of the sequence of events in a trial $(A)$ and the timeline of the experiment $(B)$

$0.3 \mu \mathrm{l}$ per $3 \mathrm{~min}$. The AP coordinate was taken from bregma, the $\mathrm{L}$ coordinate from the midline, and the DV coordinate from dura.

Data analysis. Analyses were conducted using SPSS for Windows (version 9.0; SPSS, Chicago, IL). The total number of choices of the large reward per delay per session (maximum 10 responses) was used to analyze choice behavior. These data were arcsine transformed to limit the effect of an artificially imposed ceiling. The number of trials omitted did not affect calculation of this measure. To determine whether animals had successfully acquired the task and reached stable baseline performance, data from seven sessions were analyzed by repeated-measures ANOVA with two within-subjects factors (DAY and DELAY). If the effect of DELAY was significant at the $p<0.05$ level but there was no main effect of DAY, animals were judged to have reliably acquired the task. The behavioral effects of the two lesions were assessed by comparing the performance of animals in each lesion group to data from sham-operated controls. Data from seven stable postoperative sessions were analyzed using repeated-measures ANOVA with LESION as a between-subjects factor and DAY and DELAY as within-subjects factors. The number of trials omitted, the latency to respond on the levers, and the latency to collect reward were also analyzed.

Histology. After the completion of behavioral testing, animals were terminally anesthetized with sodium pentobarbitone $(3 \mathrm{ml} / \mathrm{kg}$, i.p.; Genus Express, Dunnington, UK) and perfused transcardially with $0.01 \mathrm{M}$ PBS followed by $4 \%$ paraformaldehyde. The brains were removed, postfixed in $4 \%$ paraformaldehyde, and transferred into 20\% sucrose in $0.01 \mathrm{M}$ PBS $24 \mathrm{hr}$ before being cut on a freezing microtome. Coronal sections $(60 \mu \mathrm{m})$ were stained with cresyl violet, and the location of the lesions was mapped onto standardized sections of the rat brain (Paxinos and Watson, 1998). 


\section{Results}

\section{Lesion analysis}

Histological verification of the size and location of lesions depicted in Figure 2 was conducted by observers who were blind to the experimental conditions and results. Five animals were excluded from the OFC lesion group because the lesions were incomplete, unilateral, or extended into the prelimbic cortex. Three animals were excluded from the BLA lesion group because lesions incorporated the central nucleus of the amygdala, extended too far rostrally, or were incomplete. In general, the behavioral profile of animals with lesions encompassing the targeted area behaved in a similar manner to those with lesions restricted to that area, but the former were omitted from the analysis.

\section{Behavioral analysis}

Preoperatively, a typical pattern of delaydependent choice behavior was observed, with animals preferring the large reward at the beginning of the session when its delivery was immediate and shifting their preference to the small reward as the delay to the large reward increased (DELAY: $\left.F_{(4,116)}=66.4 ; p<0.0001\right)$ (Fig. 3).

Postoperatively, animals with BLA lesions $(n=7)$ preferred the small immediate reward to a greater extent than shamoperated controls (DELAY $\times$ LESION: $\left.F_{(4,240)}=3.346 ; p<0.017\right)$, whereas OFClesioned rats $(n=7)$ showed the opposite pattern of behavior, increasing their choice of the larger delayed reward (DELAY $\times$ LESION: $F_{(4,240)}=3.156 ; p<$ 0.024 ) (Fig. 3). When the delays were removed, all animals consistently chose the large reward, indicating that subjects in each group were capable of discriminating relative reward magnitude. Animals with BLA or OFC lesions did not omit more trials compared with sham-operated controls (LESION: $F_{(2,20)}=0.231$; NS), and the latencies to respond and to collect reward were similar in all three groups (response latency, LESION: $F_{(2,20)}=1.51$, NS; collection latency, LESION: $\left.F_{(2,20)}=0.233, \mathrm{NS}\right)($ Table 1$)$.

\section{Discussion}

Although selective damage to the BLA increased impulsivity in a rodent delay-discounting task, OFC lesions decreased impulsivity. The contrasting nature of these changes provides perhaps the first behavioral evidence of a dissociation between the functional role of these two areas in modulating cognition and also delineates the neural circuitry involved in mediating this form of impulsivity.

\section{BLA lesions increase impulsive choice: impairments in maintaining representations of reward}

Theoretically, BLA lesions may have increased impulsive choice by preventing the animal from remembering that responding on lever
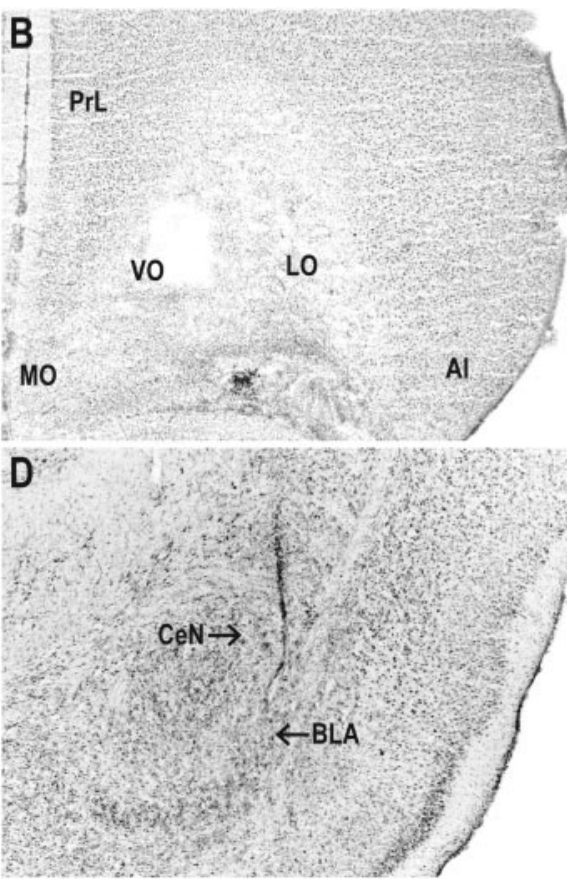

Figure 2. $A-D$, Photomicrographs depicting a typical OFC lesion $(B)$ and BLA lesion $(D)$ compared with appropriate sham control tissue ( $A$ and $C$, respectively). M0, Medial orbital cortex; V0, ventral orbital cortex; LO, lateral orbital cortex; Al, agranular insular cortex; CeN, central amygdaloid nucleus.
PRE-OP

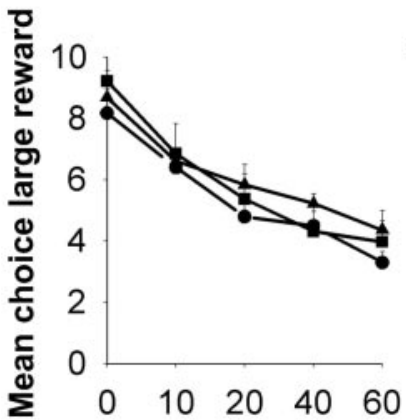

POST-OP

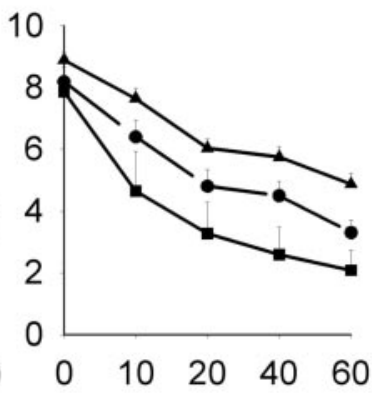

POST-OP: NO DELAYS

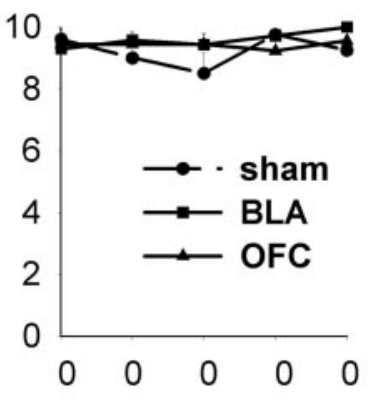

\section{Delay to large reward per block (s)}

Figure 3. Effect of lesions to the OFC and BLA on performance of a delay-to-gratification task compared with sham-operated controls. Data shown are mean number of responses and SEM taken from seven consecutive postoperative sessions.

B leads to delivery of the large reward when it is delayed. Few studies have directly investigated whether BLA lesions impair conditioning with long CS- unconditioned stimulus (US) intervals, although blockade of $\mathrm{GABA}_{\mathrm{A}}$ receptors in the BLA facilitates, rather than impairs, aversive learning with CS-US delays (Ferry and Di Scala, 1997). However, memories for reward and reward-predicting stimuli can be formed and used after BLA lesions, as indicated by the lack of impairment produced by these lesions on autoshaping paradigms (Parkinson et al., 2000). Because BLA-lesioned rats strongly preferred the large reward in the absence of delays, it is unlikely that impulsive choice occurred simply because the large reward lacked value under all conditions. The increase in impulsivity may also have reflected an increased sensitivity to satiation in BLA-lesioned rats (and vice versa in OFC-lesioned animals). Although possible, previous work has indicated that changes in satiation do not significantly alter delay-discounting in trained animals (Cardinal et al., 2000).

Alternatively, increased impulsive choice after BLA lesions 
Table 1. Levels of omissions, latency to respond, and latency to collect reward per session

\begin{tabular}{|c|c|c|c|c|c|c|}
\hline \multirow[t]{2}{*}{ Variable } & \multicolumn{3}{|c|}{ Preoperative } & \multicolumn{3}{|c|}{ Postoperative } \\
\hline & Sham & BLA & OFC & Sham & BLA & OFC \\
\hline Omissions & $0.34(0.30)$ & $0.28(0.30)$ & $0.37(0.28)$ & $0.69(0.56)$ & $0.50(0.33)$ & $0.35(0.17)$ \\
\hline Latency to respond (sec) & $1.01(0.07)$ & $0.96(0.07)$ & $0.92(0.04)$ & $1.19(0.16)$ & $0.99(0.05)$ & $1.22(0.09)$ \\
\hline Latency to collect reward (sec) & $0.29(0.01)$ & $0.29(0.03)$ & $0.32(0.04)$ & $0.29(0.14)$ & $0.23(0.07)$ & $0.32(0.03)$ \\
\hline
\end{tabular}

Data shown are mean (SEM).

may reflect an impairment in using representations of the incentive value of reward to guide behavior. To maintain responding for the large reward when it is delayed, subjects may hold a representation of the reward "on-line" during the delay period. Responding on the lever leading to delivery of the large reward, or the postresponse delay itself, can function as conditioned reinforcers, thus helping to maintain responding despite the delay to gratification (Garrud et al., 1981).

The BLA is critically involved in such conditioned reinforcement processes through maintaining a representation of the appetitive value of conditioned stimuli (Cador et al., 1989; Burns et al., 1993; Gallagher, 2000), and there is considerable behavioral evidence suggesting that interactions between the BLA and NAC are important in enabling such stimuli to guide behavior (Cardinal et al., 2002; Setlow et al., 2002). In this study, BLA lesions may have prevented the utilization of such representations. Therefore, the value of the large reward was not maintained across the delay, leading the subject to choose impulsively. The NAC has been described as the "limbicmotor interface," providing an important route whereby the limbic system influences actions through allowing CSs to guide behavior (Mogenson et al., 1980). The fact that similar increases in impulsive choice are observed after NAC lesions indicates that the NAC and BLA may be part of a common circuit promoting choice of the large delayed reward, potentially through maintaining a representation of such a reward in its absence.

\section{OFC lesions decrease impulsive choice: impairments in updating representations of incentive value on the basis of consequences of a response}

The decrease in impulsive choice caused by OFC lesions contrasts with the comparative lack of effect of PrL or ACC lesions on this paradigm. Dissociable effects after damage to different frontal regions have been observed in numerous behavioral tests, leading to the suggestion that dorsomedial prefrontal cortex is integral to attentional and mnemonic processes, whereas ventral prefrontal regions such as OFC enable cognitive and behavioral flexibility (Robbins, 1996; Bechara et al., 1998; Chudasama et al., 2003). In particular, subjects with OFC lesions perseverate in responding to the previously rewarded stimulus during reversal learning (Jones and Mishkin, 1972; Chudasama and Robbins, 2003). Decreased impulsive choice after OFC lesions may therefore arise through perseveration in the response associated with the largest reward, regardless of the consequences of that response.

Although neurons encoding reinforcement are found throughout the PFC, dissociations have been observed between the firing pattern of neurons within the primate dorsolateral and orbitofrontal PFC in response to CSs indicative of a more primary role for the OFC in representing reward-related information (Wallis and Miller, 2003). The fact that OFC lesions increase responding for the large delayed reward and increase perseverative behavior supports the view that the OFC is involved in revising the representation of the incentive value of a response as the outcome associated with that response change (Schoenbaum et al., 2003a). As the delay to the large reward increases, responding for that reward becomes less attractive as the delay decreases the subjective value of the large reward (Ainslie, 1975). OFC-lesioned rats did not show such a marked shift toward preferring the smaller immediate reward, indicating that the delay failed to sufficiently devalue the large reward, and that the change in reinforcement contingency had minimal impact on decision-making processes.

The finding that OFC lesions reduced impulsive choice seemingly contradicts the observation that patients with OFC damage choose more impulsively in gambling paradigms (Bechara et al., 1999; Rogers et al., 1999). However, increased choice of risky options in gambling tasks and increased choice of the large delayed reward in the present study may both result from inadequate integration of the consequences of responding for the large reward with the incentive value of that reward, so that the anticipated punishment (loss of money in the former task, delay to reward delivery in the latter) ultimately fails to sufficiently devalue the response outcome.

\section{Related yet dissociable roles for the BLA and OFC in regulating goal-directed behavior}

These data add to a growing body of evidence suggesting that, although the BLA and OFC cooperate to regulate goal-directed behavior (Baxter et al., 2000), the BLA primarily encodes the incentive value of a stimulus, whereas the OFC is also implicated in monitoring and updating representations of expected outcomes associated with making responses on the basis of such stimuli. This distinction is supported by electrophysiological recordings in the BLA and OFC obtained during performance of an odor-mediated go/no-go discrimination task (Schoenbaum et al., 1999). Although neuronal firing in the BLA primarily encodes the current motivational significance of these stimuli, the firing pattern of OFC neurons signals the specific sensory properties of the stimulus in addition to its predictive value. Furthermore, the firing of OFC neurons is more indicative of the current behavioral strategy, particularly when the outcome predicted by a stimulus is altered or reversed (Schoenbaum et al., 2000). A previous study has shown that lesions to the OFC performed before the acquisition of a delay-discounting task have the opposite effect, increasing choice of the small immediate reward (Mobini et al., 2002). This accords with observations that OFC neurons encoding the expected outcome of a response are only observed late in training once accurate performance has been acquired, unlike neuronal activity in the BLA (Schoenbaum et al., 1999). Removing the modulatory influence of the OFC before and after the acquisition of reward contingencies may thus have very different effects on choice behavior.

Because the BLA and OFC are heavily interconnected, the behavioral changes caused by damage to one region could arise in part from disrupted functioning in the other. Recently, it has been demonstrated that lesions to the BLA alter the response properties of neurons in the OFC during go/no-go performance, 
so that cues predicting reward delivery fail to activate neurons encoding the expected outcome in this region (Schoenbaum et al., 2003b). Hence, the fact that BLA lesions decreased choice of the large delayed reward could reflect alterations in the capability of the OFC to use information maintained in the BLA regarding cues predictive of reward in the task (i.e., responding on the lever associated with reward delivery) to strengthen the representation of the expected outcome (delivery of the large reward).

In summary, current findings implicate pathways involving the OFC, BLA, and NAC core in mediating impulsive choice, whereas input from the PrL and ACC does not play a critical role. Clearly, different frontal regions make relatively specific contributions to the regulation of behavior despite gross similarities in their efferent and afferent projections (Öngür and Price, 2000; Pitkänen, 2000). Such functional dissociations may reflect differences in the precise topographical organization of frontostriatal projections, in which a ventral-to-dorsal gradient in the PFC corresponds to a ventromedial-to-dorsolateral gradient in the striatum (Berendse et al., 1992). The finding that lesions to the OFC and BLA can shift the delay-discounting function in opposite directions also delineates the relative contributions of these two areas in the regulation of goal-directed behavior and impulsive decision making.

\section{References}

Ainslie G (1975) A behavioral theory of impulsiveness and impulse control. Psychol Bull 82:463-498.

Baxter MG, Parker A, Lindner CCC, Izquierdo AD, Murray EA (2000) Control of response selection by reinforcer value requires interaction of amygdala and orbital prefrontal cortex. J Neurosci 20:4311-4319.

Bechara A, Damasio H, Tranel D, Anderson SW (1998) Dissociation of working memory from decision making within the human prefrontal cortex. J Neurosci 18:428-437.

Bechara A, Damasio H, Damasio AR, Lee GP (1999) Different contributions of the human amygdala and ventromedial prefrontal cortex to decisionmaking. J Neurosci 19:5473-5481.

Berendse HW, Galis-de Graaf Y, Groenewegen HJ (1992) Topographical organization and relationship with ventral striatal compartments of prefrontal corticostriatal projections in the rat. J Comp Neurol 316:314-347.

Burns LH, Robbins TW, Everitt BJ (1993) Differential effects of excitotoxic lesions of the basolateral amygdala, ventral subiculum and prefrontal cortex on responding with conditioned reinforcement and locomotor activity potentiated by intra-accumbens infusions of amphetamine. Behav Brain Res 55:167-183.

Cador M, Robbins TW, Everitt BJ (1989) Involvement of the amygdala in stimulus-reward associations: interaction with the ventral striatum. Neuroscience 30:77-86.

Cardinal RN, Robbins TW, Everitt BJ (2000) The effects of d-amphetamine, chlordiazepoxide, alpha-flupenthixol and behavioral manipulations on choice of signalled and unsignalled delayed reinforcement in rats. Psychopharmacology 152:362-375.

Cardinal RN, Pennicott DR, Sugathapala CL, Robbins TW, Everitt BJ (2001) Impulsive choice induced in rats by lesions of the nucleus accumbens core. Science 292:2499-2501.

Cardinal RN, Parkinson JA, Hall J, Everitt BJ (2002) Emotion and motivation: the role of the amygdala, ventral striatum, and prefrontal cortex. Neurosci Biobehav Rev 26:321-352.

Chudasama Y, Robbins TW (2003) Dissociable contributions of the orbitofrontal and infralimbic cortex to pavlovian autoshaping and discrimination reversal learning: further evidence for the functional heterogeneity of the rodent frontal cortex. J Neurosci 23:8771-8780.

Chudasama Y, Passetti F, Rhodes SEV, Lopian D, Desai A, Robbins TW (2003) Dissociable aspects of performance on the 5-choice serial reaction time task following lesions of the dorsal anterior cingulae, infralimbic and orbitofrontal cortex in the rat: differential effects on selectivity, impulsivity and compulsivity. Behav Brain Res 146:105-119.

Damasio AR (1994) Descartes' error. New York: Putman.
Evenden JL, Ryan CN (1996) The pharmacology of impulsive behavior in rats: the effects of drugs on response choice with varying delays of reinforcement. Psychopharmacology 128:161-170.

Ferry B, Di Scala G (1997) Bicuculline administration into basolateral amygdala facilitates trace conditioning of odor aversion in the rat. Neurobiol Learn Mem 67:80-83.

Gallagher M (2000) The amygdala and associative learning. In: The amygdala: a functional analysis, Ed 2 (Aggleton JP, ed), pp 311-330. New York: Oxford UP.

Garrud P, Goodall G, Mackintosh NJ (1981) Overshadowing of a stimulusreinforcer association by an instrumental response. J Exp Psychol 33B:123-135.

Jones B, Mishkin M (1972) Limbic lesions and the problem of stimulusreinforcement associations. Exp Neurol 36:362-377.

Krettek JE, Price JL (1977) Projections from the amygdaloid complex to the cerebral cortex and thalamus in the rat and cat. J Comp Neurol 172:687-722.

LeDoux JE (2000) The amygdala and emotion: a view through fear. In: The amygdala: a functional analysis, Ed 2 (Aggleton JP, ed), pp 289-310. New York: Oxford UP.

Logue AW (1988) Res on self-control-an integrating framework. Behav Brain Sci 11:665-709.

McDonald AJ (1991) Organization of amygdaloid projections to the prefrontal cortex and associated striatum in the rat. Neuroscience 44:1-14.

Mobini S, Body S, Ho M-Y, Bradshaw CM, Szabadi E, Deakin JFW, Anderson IM (2002) Effects of lesions of the orbitofrontal cortex on sensitivity to delayed and probabilistic reinforcement. Psychopharmacology 160:290-298.

Mogenson GJ, Jones DL, Yim CY (1980) From motivation to action: functional interface between the limbic system and the motor system. Prog Neurobiol 14:69-97.

Nauta WJH (1971) The problem of the frontal lobe: a reinterpretation. J Psychiat Res 8:167-187.

Öngür D, Price JL (2000) The organization of networks within the orbital and medial prefrontal cortex of rats, monkeys and humans. Cereb Cortex 10:206-219.

Parkinson JA, Robbins TW, Everitt BJ (2000) Dissociable roles of the central and basolateral amygdala in appetitive emotional learning. Eur J Neurosci 12:405-413.

Paxinos G, Watson C (1998) The rat brain in stereotaxic co-ordinates, Ed 4. Sydney: Academic.

Pitkänen A (2000) Connectivity of the rat amygdaloid complex. In: The amygdala: a functional analysis, Ed 2 (Aggleton JP, ed), pp 31-116. New York: Oxford UP.

Robbins TW (1996) Dissociating executive functions of the prefrontal cortex. Phil Trans R Soc Lond B Biol Sci 351:1463-1471.

Rogers RD, Everitt BJ, Baldacchino A, Blackshaw AJ, Swainson R, Wynne K, Baker NB, Hunter J, Carthy T, Booker E, London M, Deakin JFW, Sahakian BJ, Robbins TW (1999) Dissociable deficits in the decision-making cognition of chronic amphetamine abusers, opiate abusers, patients with focal damage to prefrontal cortex, and tryptophan-depleted normal volunteers: evidence for monoaminergic mechanisms. Neuropsychopharmacol 20:322-339.

Schoenbaum G, Chiba AA, Gallagher M (1999) Neural encoding in orbitofrontal cortex and basolateral amygdala during olfactory discrimination learning. J Neurosci 19:1876-1884.

Schoenbaum G, Chiba AA, Gallagher M (2000) Changes in functional connectivity in orbitofrontal cortex and basolateral amygdala during learning and reversal learning. J Neurosci 20:5179-5189.

Schoenbaum G, Setlow B, Ramus SJ (2003a) A systems approach to orbitofrontal cortex function: recordings in rat orbitofrontal cortex reveal interactions with different learning systems. Behav Brain Res 146:19-29.

Schoenbaum G, Setlow B, Saddoris MP, Gallagher M (2003b) Encoding predicted outcome and acquired value in orbitofrontal cortex during cue sampling depends upon input from basolateral amygdala. Neuron 39:855-867.

Setlow B, Holland PC, Gallagher M (2002) Disconnection of the basolateral amygdala complex and nucleus accumbens impairs Pavlovian secondorder conditioned responses. Behav Neurosci 116:267-275.

Wallis JD, Miller EK (2003) Neuronal activity in primate dorsolateral and orbital prefrontal cortex during performance of a reward preference task Eur J Neurosci 18:2069-2081. 